\title{
The temporal structure of spike trains in the primate basal ganglia: afferent regulation of bursting demonstrated with precentral cerebral cortical ablation
}

\author{
J. Wayne Aldridge and Sid Gilman \\ Department of Neurology, University of Michigan, Ann Arbor, MI 48104-1687 (U.S.A.)
}

(Accepted 2 October 1990)

Key words: Putamen; Caudate nucleus; Neostriatum; Single unit; Bursting; Primate; Ablation; Motor cortex

\begin{abstract}
We studied the temporal pattern of discharge of single units in the basal ganglia of awake primates sitting quietly. Bursting was studied with a procedure that identified individual bursts in a spike train, quantifying burst intensity (surprise), bursts per 1000 spikes, spikes per burst and burst length. Autocorrelation techniques were used to assess the dependencies of spike trains on the temporal order of intervals. Striatal units had a greater tendency to burst (79\% of units) than pallidal units $(50 \%)$. The caudate nucleus and putamen had nearly identical burst properties on all measures. In the pallidum, bursting was more prevalent in the external segment and bursts were more intense and more frequent than in the internal segment. The autocorrelation analysis revealed that the temporal structure of the spike train was more dependent on the order of intervals in the striatum than in the pallidum. Bursting units had an increased probability of discharge after each spike and the relative refractory period was shorter in bursting units than units without bursts. Very few units exhibited cyclic discharge properties. Ablations of areas 4 and 6 in the precentral cortex demonstrated that striatal bursting was under afferent control. The putamen, which receives more cortical afferents from areas 4 and 6 than the caudate nucleus, had fewer and less intense bursts after the afferents were lesioned. Burst intensity did not change in the pallidum after the lesion. The findings indicate that bursting properties contribute to discharge variability in the basal ganglia and suggest that information transfer in the striatum may utilize bursts. In contrast, rate coding may be a more important mechanism for units in the pallidum.
\end{abstract}

\section{INTRODUCTION}

Neuronal activity patterns can play an important part in determining the integrative functions of the central nervous system. Pathologically induced changes in neuronal activity may account for disturbed function. In the basal ganglia, changes in the rate of neuronal activity due to degenerative processes or lesions are thought to explain the behavioral and motor disturbances associated with damage to these structures ${ }^{2,47}$. Most proposals for function and dysfunction in the basal ganglia are concerned with information transfer coded through discharge frequency. But frequency coding is only one of many potential information coding possibilities ${ }^{33,34}$. Alternative coding mechanisms depend on pattern, that is, the temporal sequence of intervals between action potentials. The pattern of intervals in a spike train can differ even though the average rate does not change. In the motor system, temporal discharge pattern is an important characteristic of corticomotoneuronal cells ${ }^{37}$ and critical to the optimum development of muscle tension ${ }^{46}$. Sensory systems also rely on discharge patterns ${ }^{35,51,52,61}$.

The anatomical and physiological substrates for a frequency coding mechanism are available within the basal ganglia. Cerebral cortical afferents increase the firing rate of striatal neurons through an excitatory ${ }^{22}$ glutamatergic pathway ${ }^{12,40,65}$. Intracellular studies of medium spiny neurons in the striatum demonstrate that discharge frequency is directly proportional to injected current, a characteristic necessary for frequency coding ${ }^{7}$. $17,30,31$. Striatal projections inhibit the pallidum through GABAergic connections $\mathbf{s}^{22,39,44}$. The pallidum may also be modulated by co-localized peptides ${ }^{23,32,48}$. Pallidal neurons with a high discharge rate are common. Pallidal discharge rate is correlated with the degree of corrective postural movements ${ }^{6}$ and is proportional to limb movement amplitude and/or velocity ${ }^{19}$. Bursting discharge has been described in neurons of the external but not the internal pallidal segments ${ }^{10}$.

Although frequency coding may occur in the basal ganglia, the temporal pattern may be equally or more important. The spontaneous discharge rate of striatal units is low and variable ${ }^{3}$, characteristics not well suited to frequency coding. Ablation of the cerebral cortical projections in areas 4 and 6 produces in striatal units an increase in spontaneous firing rate accompanied by a

Correspondence: J. Wayne Aldridge, University of Michigan, 1103 East Huron, Ann Arbor, MI 48104-1687, U.S.A. 
reduction in discharge variability ${ }^{3}$. These findings suggest that the pattern of striatal discharge is important in this pathway and that it is under afferent control ${ }^{3}$. Analogous findings on pallidal discharge pattern are observed after lesions or pharmacologic manipulation of striatopallidal projections $^{14,45,54}$ and alterations in the temporal structure of pallidal output in response to striatal stimulation is associated with MPTP-induced parkinsonism ${ }^{57}$. Thus, the temporal sequence or pattern of spikes may be an important property of all basal ganglia neurons. Although the importance of discharge pattern is indicated by the changes in variability, little is revealed about the underlying temporal structure of the spike trains from measures of variability alone. Bursting, cyclic discharge and repeating patterns may all contribute. Bursting patterns are anticipated since they have been described in both the striatum and the pallidum ${ }^{10.18,64}$.

The current study was initiated to assess quantitatively the temporal discharge properties of spontaneous spike trains in the neostriatum and pallidum. The effects of afferent inputs on the temporal patterns were examined by ablation of precentral cortical areas 4 and 6 . A new technique to identify bursts and characterize their properties $^{35}$ was used.

\section{MATERIALS AND METHODS}

\section{Animals}

This study extends the analysis of $4 M$. fascicularis monkeys, two lesioned animals and two controls, reported previously ${ }^{3}$. The animals were prepared for chronic recording of single unit activity from the putamen, caudate nucleus, and globus pallidus of the left cerebral hemisphere. Under general anesthesia with intravenous pentobarbital, the animals were placed in a stereotaxic frame and a stainless steel recording chamber was fixed to the skull with bone screws and dental acrylic. In the lesioned animals, chamber implantation was preceded by subpial resection of areas 4 and 6 of Brodmann in the left cerebral hemisphere ${ }^{11,21}$.

\section{Recording}

Spontaneous neuronal activity was recorded while the monkeys were awake and sitting quietly in a primate chair placed inside a sound-restricted recording booth. A hydraulic microdrive was placed on the recording chamber and used to manipulate tungsten electrodes to the desired recording sites in the basal ganglia. Preamplified neuronal signals were led to an oscilloscope, audio monitor, and tape recorder. Single units were isolated and the neuronal activity was recorded on tape and digitized off-line. In one control monkey, spikes were digitized on-line, making off-line digitization of tape records unnecessary ${ }^{4}$.

Data collection began when the animals were sitting quietly. To ensure that comparisons of spontaneous unit activity were made only during quiet sitting, recordings were edited off-line to select periods without movement. Because the basal ganglia are involved in motor control, it was necessary to ensure that bursting properties were not due to movements. It is possible that some movements may have escaped detection; however, it is highly improbable that undetected movements produced systematic differences that could account for the results in such a large sample of units.

All recordings were made in the period from 8 to 60 days after the lesion. In control animals recordings were spaced over several months. On completion of recording, small electrolytic lesions were made to mark the recorded regions. The brains were frozen, sectioned, and stained. Recording tracks were reconstructed and sites of recordings were plotted ${ }^{3}$.

\section{Analysis}

Digitized spike trains were analyzed in detail off-line. Units were discriminated from each other and from background noise by a procedure comparing the negative peak, positive peak, and peak to peak time of each recorded waveform ${ }^{58}$. When two units could be discriminated adequately, both were studied; rarely, three units were discriminable. If more than one unit was present but the waveforms were not separable, the data were discarded. The mean, median, standard deviation and coefficient of variation (mean/ standard deviation) were determined for each unit. Interspike interval histograms and autocorrelation functions ${ }^{41,50}$ were computed and plotted for each unit.

For analysis of bursts, the surprise method of Legendy and Salcman ${ }^{35}$ was implemented. In this computerized procedure, the intervals in each spike train were scanned successively and periods with short intervals between spikes, i.e. bursts, were extracted. The iterative procedure compared the mean interval size in each burst to the mean interval size of the whole spike train. To be classified as a burst, the intraburst interval size had to be less than one half of the average in the whole spike train. At least two intervals (3 spikes) were needed to make a burst. For each burst selected, the Poisson surprise value, which is the negative logarithm of the probability of the occurrence of a burst in a random (Poisson) spike train $^{35}$, was calculated. A minimum surprise value of 3 (a probability value of 1 in 1000) was required for acceptance. The burst detection procedure attempted to maximize the surprise value of the burst by adding the next interval in the spike train to the burst and recomputing a new surprise value. If the surprise value increased by the addition of the next interval, it was retained in the burst and the process was repeated until the surprise value ceased to increase. When adding spikes no longer increased the surprise value, the possibility of further maximization by removing intervals from the beginning of the spike train was attempted. For each unit several properties of bursts were determined, including: the average surprise value, burst duration, number of spikes per burst, number of bursts per 1000 spikes, and intraburst rate. Both the surprise (a measure of unexpectedness) and the number of bursts per 1000 spikes give some indication of the intensity of bursts in a spike train.

Because these two measures of burst intensity could and did vary independently, an index of bursting was computed that took both of these measures into account. This index facilitated comparisons of burst strength between different units. The index was computed by calculating the square root of the product of the surprise value and the burst rate per 1000 spikes. All data items were stored in a computerized data base. Statistical analyses were done on data extracted from the data base with Systat (Systat, Inc.) and plotted using Sygraph (Systat, Inc.). Statistics were computed and comparisons between control and lesioned animals were made for each structure. The significance of the comparisons was assessed with the Mann-Whitney $U$-test unless otherwise noted.

\section{RESULTS}

The discharge properties of 500 spontaneously active units in the striatum and 366 units in the pallidum were studied. 79\% (394/500) of striatal units (Fig. 1) and 50\% $(182 / 366)$ of pallidal units (Fig. 2) had bursts. The putamen and caudate nucleus had similar proportions of burst units (Table I). In the globus pallidus, however, a larger proportion of units in the external segment had bursts than in the internal segment (Table $\mathrm{I}$ ). 


\section{Burst properties}

The quantitative properties of bursts were assessed for every unit (Figs. 1 and 2, Table II). The intensity of bursting is reflected in both the surprise value and the number of bursts per 1000 spikes. The distributions of these two measures were continuous and skewed (Fig. 3A,D,E). Typically, surprise and bursts per 1000 spikes were uncorrelated in individual units (Fig. 3B). For this reason, a single index of bursting intensity was computed from the values for surprise and bursts per 1000 spikes to facilitate between-unit comparisons (see Materials and Methods). Bursting intensity was higher in the striatum than the pallidum (Fig. 3C), with both a higher bursting rate per 1000 spikes (Fig. 3E) and higher surprise values (Fig. 3D). The distributions of burst intensity were more restricted in the pallidum (Fig. 3D,E).

The putamen was not different from the caudate nucleus in surprise values $(P=0.490)$, burst rate per 1000 spikes $(P=0.589)$ or burst index $(P=0.951)$. There was no difference between the two pallidal segments in the surprise values $(P=0.077)$, but the burst rate per 1000 spikes $(P=0.008)$ and the burst index $(P=0.008)$ values were higher in the external than the internal pallidal segment. Although burst strength parameters were consistently lower in the pallidum than the striatum, the number of bursts per unit of time (Fig. $3 \mathrm{H}$ ) and the intraburst firing rate (Fig. 3I) were higher in the globus pallidus.

The caudate nucleus was not different from the putamen in the intraburst rate $(P=0.974)$, spikes per burst $(P=0.222)$ or burst length $(P=0.406)$. The number of spikes per burst was similar in the pallidum and the striatum (Fig. 3G), but the length of the average burst was shorter in the pallidum (Fig. 3F). In comparisons between the two pallidal segments, all measures revealed significant differences. The external segment had more spikes per burst $(P<0.017)$, longer bursts $(P$ $<0.019)$ and a lower intra-burst firing rate $(P<0.037)$ than the internal segment.

In the striatum, bursting intensity and the variability of discharge as measured by the coefficient of variation were strongly correlated (Fig. 4A), that is, units with high levels of variability had high levels of bursting. In contrast, the median interval size and bursting intensity were unrelated (Fig. 4B). A weaker relationship was observed in the pallidum (Fig. 4C,D).

\section{Comparison of burst and non-burst units}

There was no difference in anatomical distribution between burst and non-burst units; both types were found evenly distributed in the striatum and pallidum.

In the external segment of the globus pallidus, the median intervals of burst and non-burst units were not
TABLE I

\section{Burst and non-burst units}

Number of units (percent of total sample).

\begin{tabular}{lrrrll}
\hline & \multicolumn{2}{l}{ Striatum } & & \multicolumn{2}{l}{ Globus pallidus } \\
\cline { 2 - 3 } \cline { 5 - 6 } \cline { 5 - 6 } & Putamen & $\begin{array}{l}\text { Caudate } \\
\text { nucleus }\end{array}$ & & $\begin{array}{l}\text { External } \\
\text { segment }\end{array}$ & $\begin{array}{l}\text { Internal } \\
\text { segment }\end{array}$ \\
\hline Burst units & $307(79 \%)$ & $87(78 \%)$ & $127(55 \%)$ & $55(40 \%)$ \\
Non-burst units & $81(21 \%)$ & $25(22 \%)$ & & $103(60 \%)$ & $81(60 \%)$ \\
Total & 388 & 112 & 230 & 136 \\
\hline
\end{tabular}

different $(P=0.37$, Fig. 5A,B), but the variability of interspike intervals was higher in burst than non-burst units $(P<0.001$, Fig. $5 \mathrm{E}, \mathrm{F})$. In the internal segment of the globus pallidus, the distributions of burst units were significantly shifted toward shorter median intervals $(P<$ 0.001 , Fig. 5A,B) and greater discharge variability $(P<$ 0.002 , Fig. $5 E, F)$ than non-burst units.

In the putamen, units with bursts had shorter median intervals $(P<0.001$, Fig. $5 \mathrm{C}, \mathrm{D})$, i.e., higher firing rates, than non-burst units. In the caudate nucleus there was no difference in median interval size $(P=0.152)$ between burst and non-burst units. The discharge variability was greater in burst than in non-burst units in both structures (caudate nucleus: $P=0.001$; putamen: $P<0.001$, Fig. $5 \mathrm{G}, 5 \mathrm{H})$.

Burst and non-burst units in the caudate nucleus were not significantly different from their counterparts in the putamen in interval size (burst units $P=0.791$, Fig. 5C; non-burst $P=0.358$, Fig. 5D) or in coefficient of variation (burst $P=0.07$, Fig. 5G; non-burst $P=0.704$, Fig. $5 \mathrm{H})$. In contrast, burst units in the external pallidal segment had greater discharge variability $(P<0.002$, Fig. 5B) than burst units in the internal segment. The median intervals of burst units did not differ in the two

\section{TABLE II}

Burst unit properties

Mean (standard error of mean).

\begin{tabular}{|c|c|c|c|c|}
\hline & \multicolumn{2}{|l|}{ Striatum } & \multicolumn{2}{|c|}{ Globus pallidus } \\
\hline & Caudate & Putamen & External & Internal \\
\hline Burst index & $14.1(0.7)$ & $13.9(0.4)$ & $6.1(0.5)$ & $4.8(0.6)$ \\
\hline $\begin{array}{l}\text { Surprise } \\
\text { Bursts/1000 }\end{array}$ & $5.9(0.3)$ & $6.3(0.2)$ & $4.2(0.1)$ & $4.3(0.3)$ \\
\hline $\begin{array}{l}\text { spikes } \\
\text { Burst length }\end{array}$ & $37.9(2.8)$ & $36.6(1.5)$ & $12.6(1.7)$ & $8.2(2.0)$ \\
\hline (ms) & $511(65)$ & $619(43)$ & $106(16)$ & $78(16)$ \\
\hline $\begin{array}{l}\text { Spikes/burst } \\
\text { Intraburst }\end{array}$ & $6.4(0.3)$ & $7.4(0.3)$ & $8.0(0.3)$ & $7.5(0.6)$ \\
\hline${ }_{n}$ rate $(\mathrm{Hz})$ & $\begin{array}{l}33.7(8.2) \\
87\end{array}$ & $\begin{array}{l}37.2(4.1) \\
307\end{array}$ & $\begin{array}{l}162.7(11.0) \\
127\end{array}$ & $\begin{array}{l}207.1(19.3) \\
55\end{array}$ \\
\hline
\end{tabular}



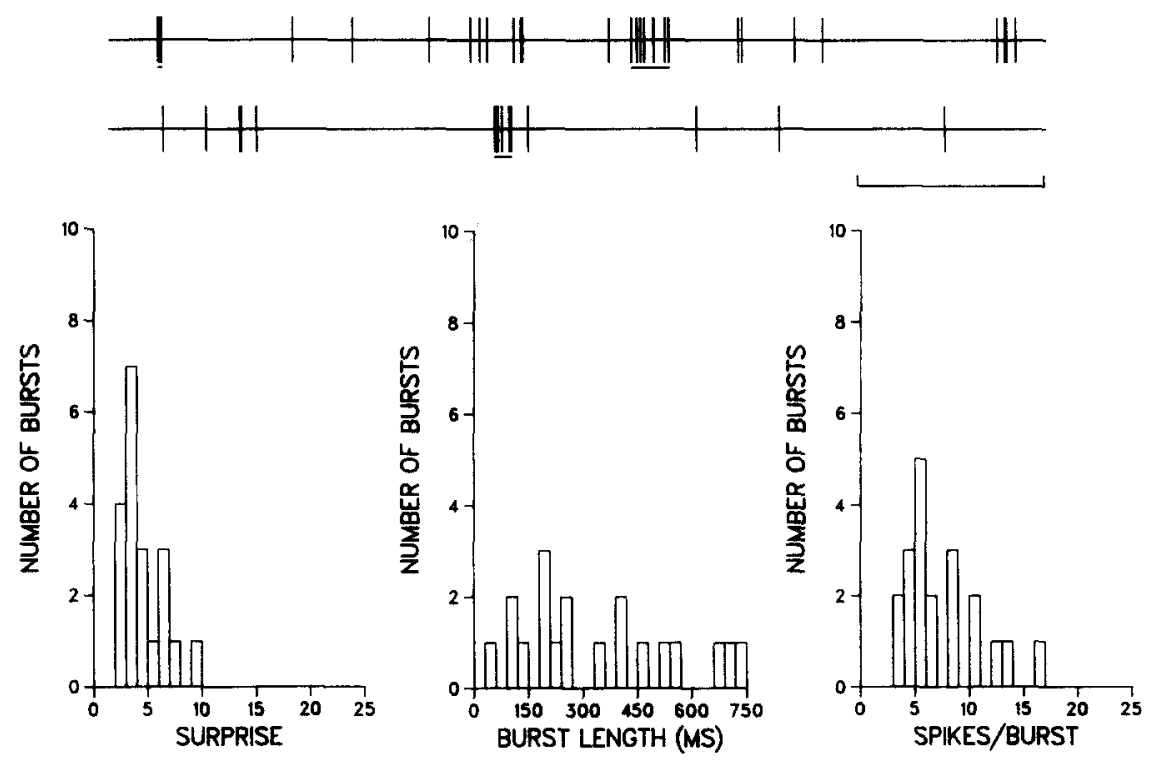

Fig. 1. Striatal burst unit. A 20-s segment from a digitized spike train of a burst unit in the putamen is shown in the top traces. The significant bursts selected by the computer algorithm are underlined. The time marker indicates $2 \mathrm{~s}$. The frequency distributions indicate the calculated surprise values, burst length and spikes per burst for this unit. The mean values for these distributions are respectively: $5.52,365 \mathrm{~ms}$, and 8 . This unit had 58 bursts per 1000 spikes and an intraburst rate of $22 \mathrm{~Hz}$. The mean burst index for this unit was 17.9. Its median discharge interval was $365 \mathrm{~ms}$ and the interspike interval coefficient of variation was 2.25 .

segments $(P=0.251$, Fig. 5A). Non-burst units in the external segment had longer intervals $(P<0.002$, Fig. 5B) and higher variability $(P<0.001$, Fig. 5F) than non-burst units in the internal pallidal segment.

A distinct subset of striatal units was identified on the basis of their low levels of bursting and discharge variability. This subset was best observed as a cluster on a plot of the coefficient of variation and median inter- spike interval size (ellipse, Fig. 6). Besides low variability and low levels of bursting, this subset was distinguished by a restricted range of intervals. Most units in this subset had median intervals between $120 \mathrm{~ms}$ and $300 \mathrm{~ms}$. Compared to the entire sample, this subset had neither short nor long median intervals. The subset overlapped the central peak in the median interval distribution (Fig. 6 ). The low variability gave the units a distinctive
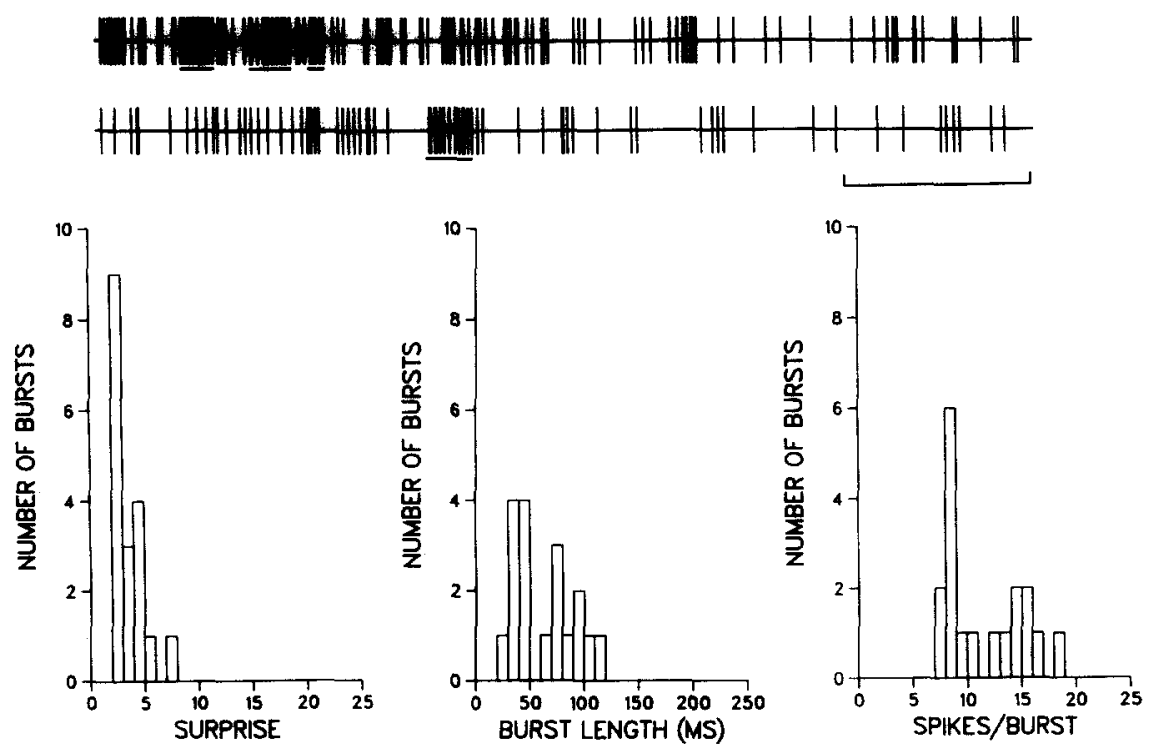

Fig. 2. Pallidal burst unit. A 5-s segment of a spike train of a burst unit in the external segment is shown. The bursts selected by the computer are underlined. The time marker represents $0.5 \mathrm{~s}$. The histograms show the distributions for surprise values (mean $=4.42$ ), burst length (mean $=72 \mathrm{~ms}$ ) and spikes per burst (mean $=11$ ). This unit had 10 bursts per 1000 spikes and an intraburst rate of $153 \mathrm{~Hz}$. The mean burst index for the unit was 6.6. Its median discharge interval was $72 \mathrm{~ms}$ and the interspike interval coefficient of variation was 4.00 . 

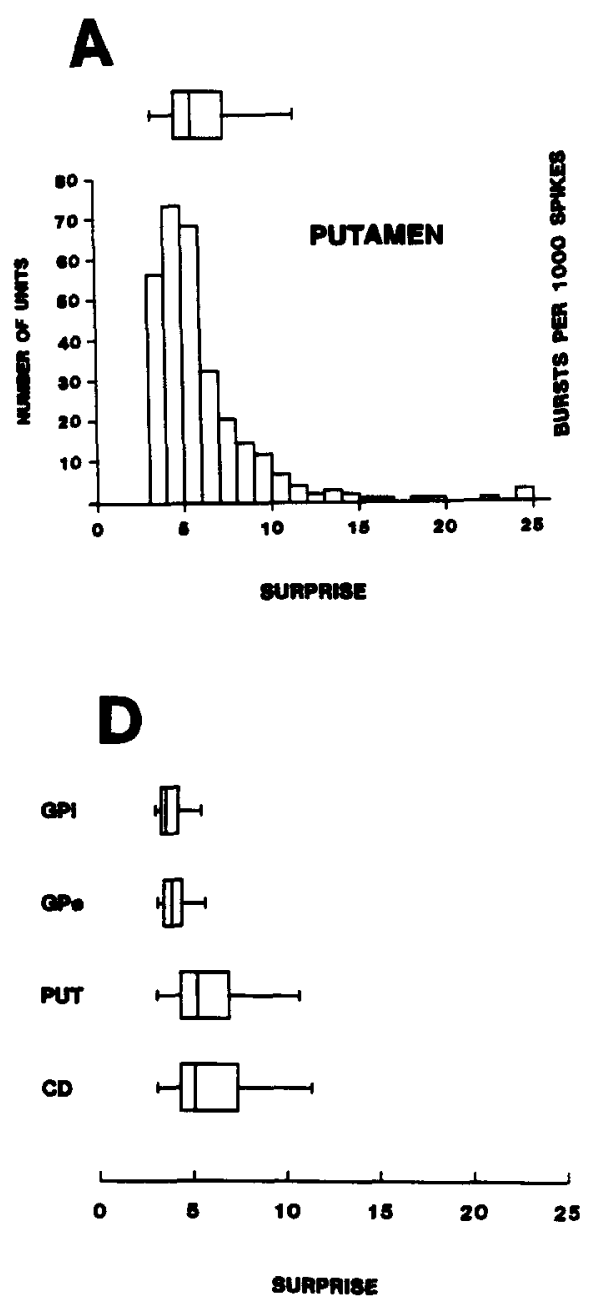

G

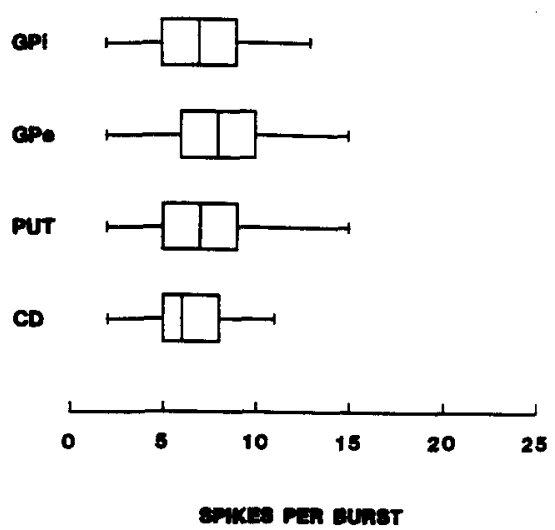

B

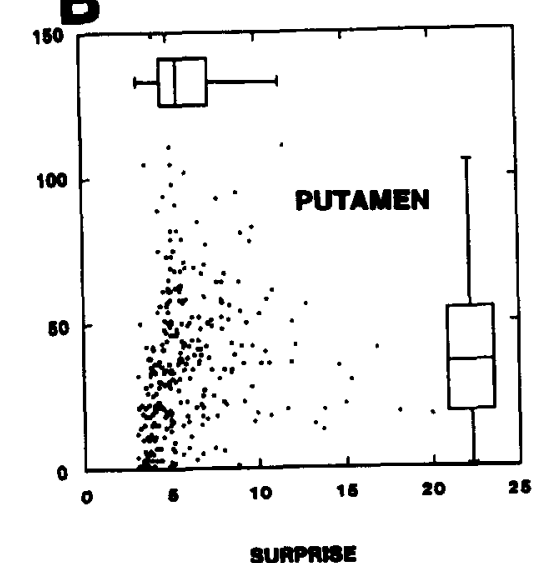

SUAPRise
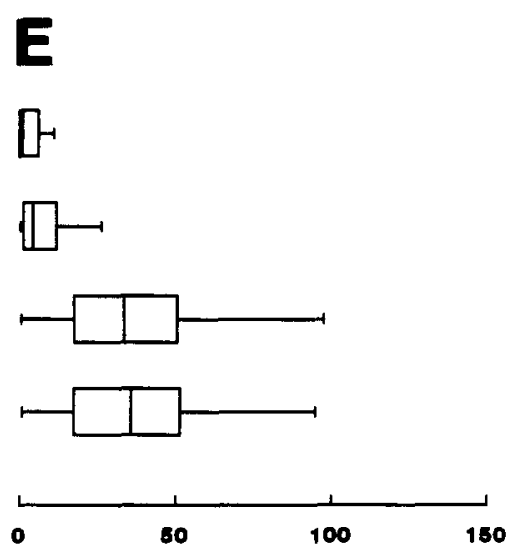

DURETS PAR 1000 eppKes

H

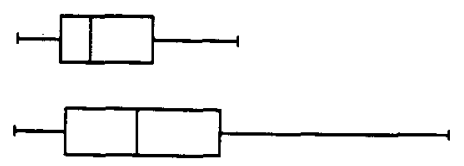

玍
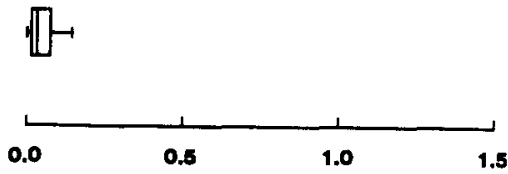

DUAST RATE (HZ)

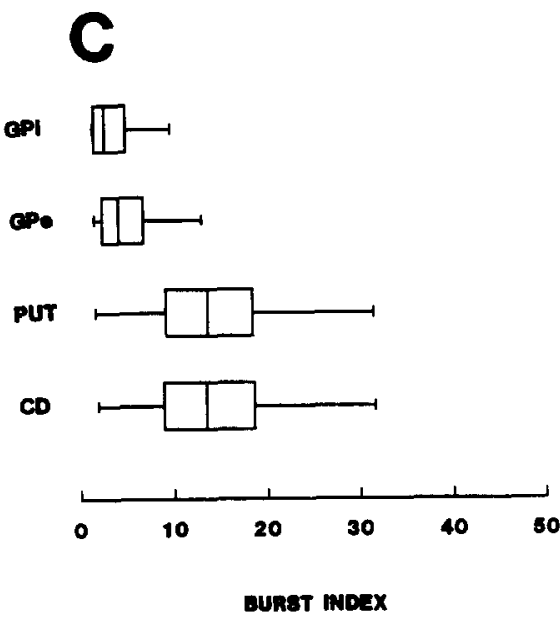

$F$

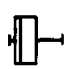

此
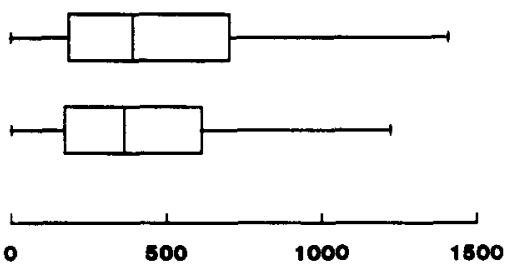

DURET LENOTH (MB)

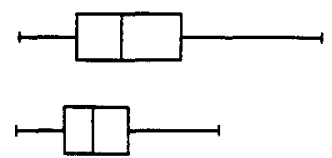

怔

足

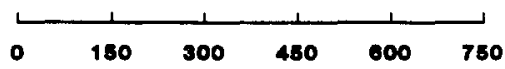

IMTRA UnET RATE (GZ)

Fig. 3. Burst properties. Part (A) illustrates the frequency distribution of Poisson surprise values for all putamen burst units. The inset box plot graphically summarizes the distribution. The median is indicated by the vertical line in the center of the box. The upper and lower vertical edges of the box indicate the midpoint between the median and the rest of the data, i.e. quartile values. The bars and tic marks extending from the box represent the distribution spread. (B) illustrates the relationship between surprise value ( $x$-axis) and bursts per 1000 spikes $(y$-axis). Parts C-I use box plots to represent the frequency distributions as in (A). Four box plots, one per structure, are aligned vertically on a common scale. (C) Burst index distributions, (D) surprise values, (E) bursts per 1000 spikes, (F) burst length, (G) spikes per burst, (H) burst rate, and (I) intraburst discharge rate. 

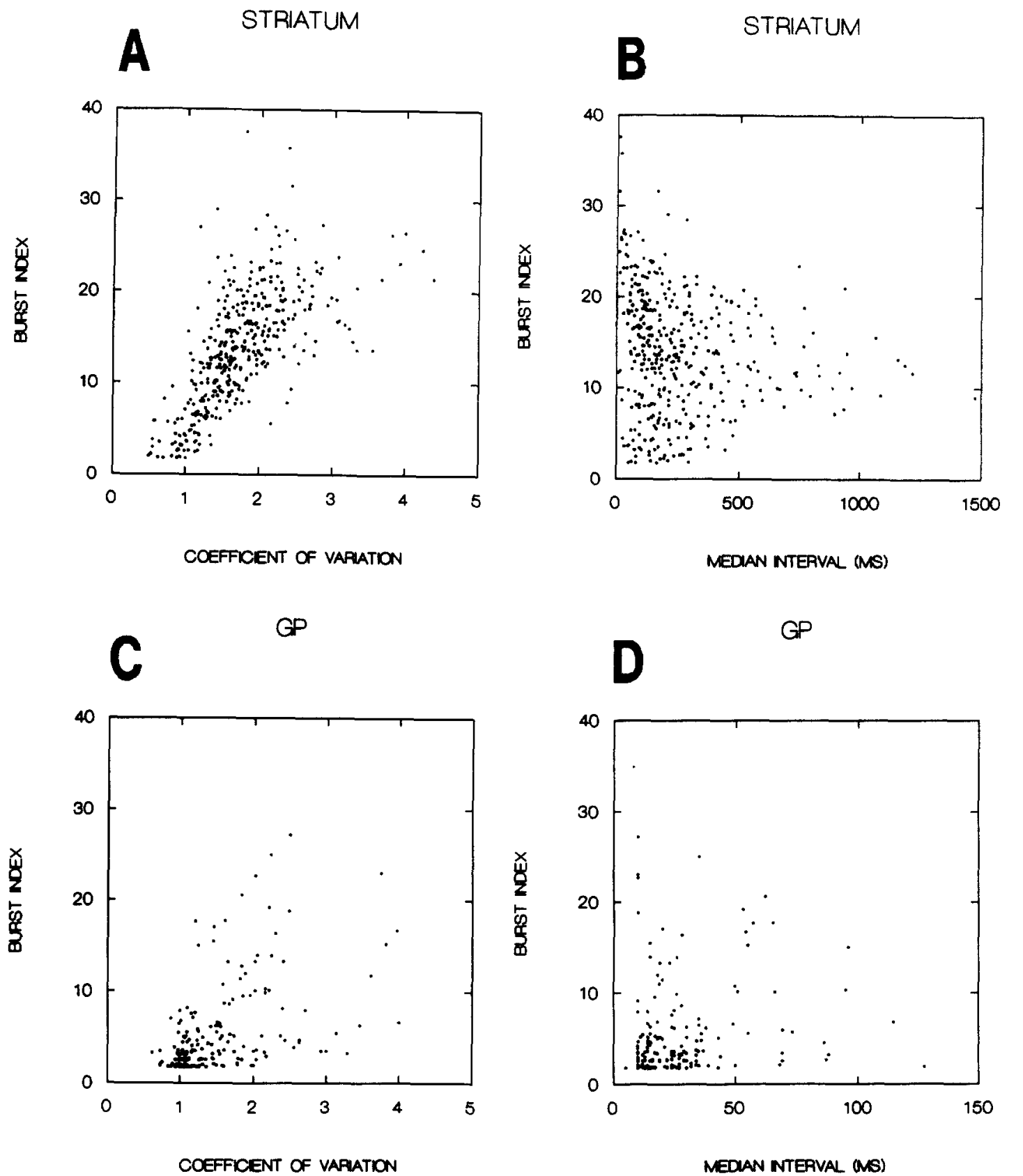

Fig. 4. Burst intensity relationships with interspike interval properties. In the striatum (A), the coefficient of variation (standard deviation/mean rate) is correlated with the burst intensity (burst index). In contrast, striatal interspike median intervals and burst index values (B) are unrelated. In (C), the relationship between coefficient of variation and burst index in the two pallidal segments is weaker than in the striatum. Part (D) illustrates the relationship between median interval size and burst index for the pallidum.

'clock-like' regularity. With a cut-off of 0.85 on the coefficient of variation axis (arrow, Fig. 6), 74 units (15\% of the striatal sample) were found in the subset. The mean firing rate was $4.6(0.3) \mathrm{Hz}$ (S.E.M.) compared to $2.6(0.2) \mathrm{Hz}$ (S.E.M.) in the whole sample. Only $28 \%$ (21/74) of the subset had bursts compared to $79 \%$ in the whole sample. The coefficient of variation values of this cluster blend into the end of the variability distribution (Fig. 6), but with median interval size taken into consideration this group is well distinguished from the rest of the population. No similar subset of units was detected in the pallidum.

\section{Autocorrelation properties}

An autocorrelation analysis was used to evaluate the dependence of the spike train on the temporal order of interspike intervals and the tendency to cyclic firing. Spike trains with 300 spikes or more were examined since fewer spikes than this produced autocorrelation plots that were too irregular for reliable evaluation. Since the 


\section{BURST NON-BURST}

A

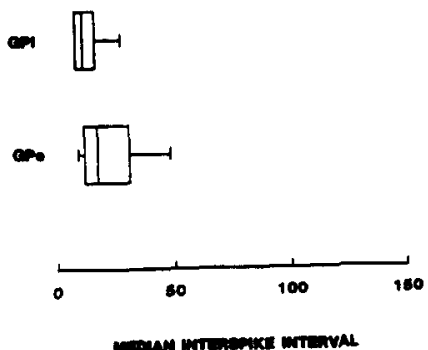

C

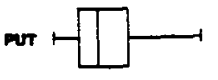

$\infty$
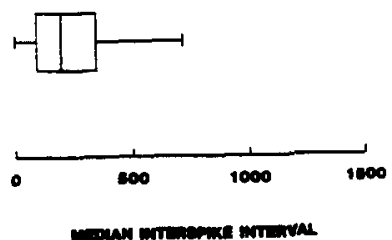

E

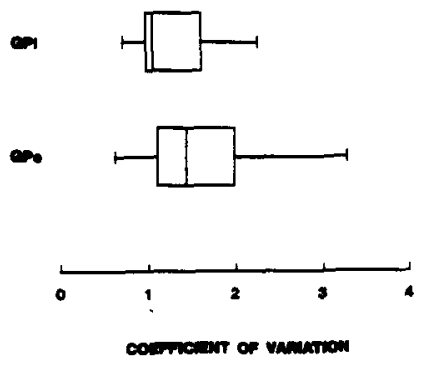

$\mathbf{G}$

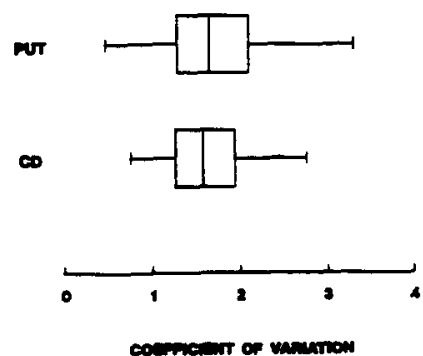

B
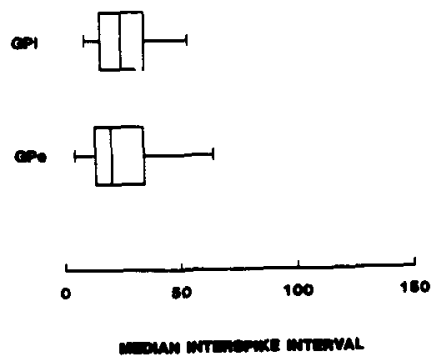

D

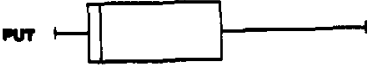

$\mathbf{c o s}$
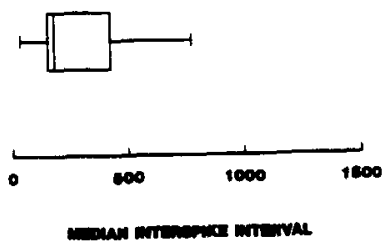

$\mathbf{F}$

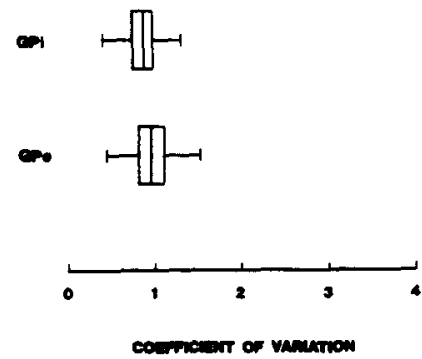

H

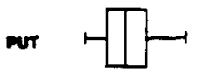

co
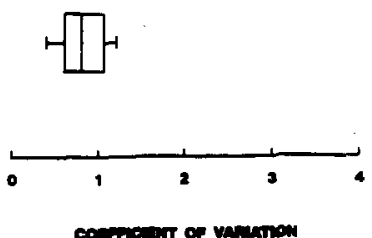

Fig. 5. Comparison of burst and non-burst units. The median interspike interval distributions represented by box plots (see format in Fig. 3A) for burst units in the pallidum (A) and striatum (C) are contrasted with non-burst units in (B) and (D) respectively. Burst units in GPi (A) have shorter intervals than non-burst units $(B)$. In the putamen, burst units $(C)$ have shorter intervals than non-burst units $(D)$. (E-H) compare interspike interval variability as measured by the coefficient of variation. Both pallidal segments exhibit more variability in burst units (E) than non-burst units (F). Likewise, in the striatum burst units are more variable (G) than non-burst units $(\mathrm{H})$. 


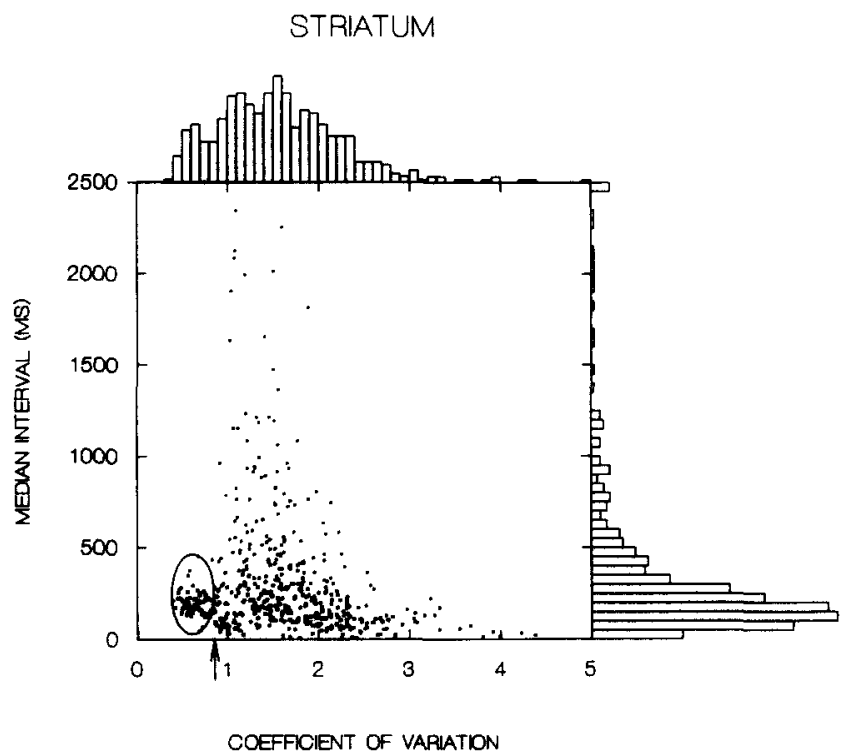

Fig. 6. The arrow indicates the empirical cut-off value $(0.85)$ on the correlation coefficient axis. The subset of units with correlation coefficients less than the cut-off is marked by the ellipse. The narrow range of discharge rates in the subset overlaps the central portion of the distribution for the whole sample (right $y$-axis). The correlation coefficient values of the subset blend smoothly into the low end of the sample distribution (upper $x$-axis).

recording time was limited, this requirement worked against slowly-firing striatal units. Nevertheless, 195 striatal units $(39 \%)$ met the criterion and, except for differences in rate, had similar properties to the whole population. Seventy-one $\%$ of striatal units meeting the criterion had bursts compared to $79 \%$ in the whole sample. $84 \%$ of pallidal units $(331 / 366)$ had sufficient spikes for an autocorrelation analysis and $49 \%$ of these units tested with autocorrelation had bursts compared to $50 \%$ in the whole sample.

Thirty-three $\%$ of striatal units $(65 / 195)$ and $81 \%$ (268/331) of pallidal units exhibited autocorrelation functions that were flat following a brief period of inactivity (Fig. 7). The early inactivity was due to the refractory

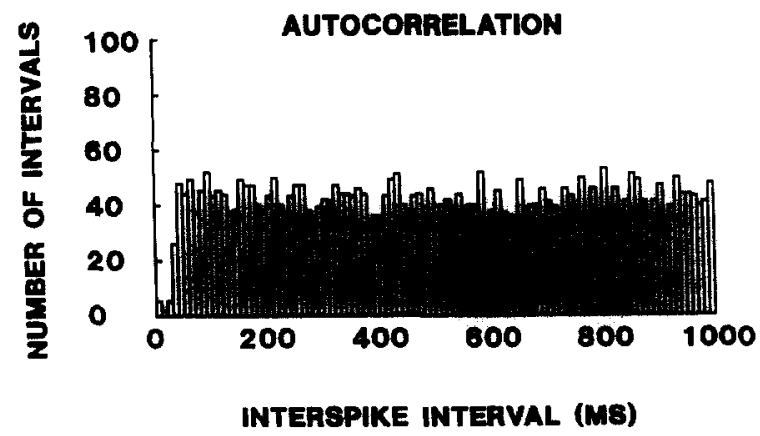

Fig. 7. The autocorrelation function of a unit from the external segment of the pallidum illustrating a flat distribution. The depression in the first 4 bins is due to the refractory properties of the unit.

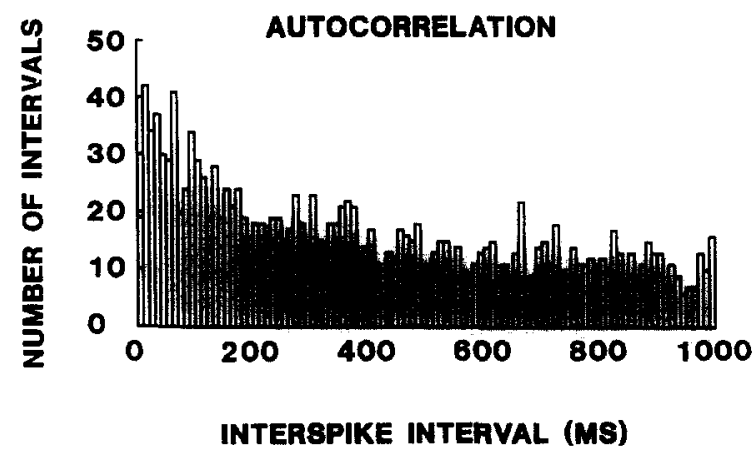

Fig. 8. Early peak autocorrelation. This unit from the putamen has an early peak in the autocorrelation function indicating that after each spike there is a period of increased probability to discharge. In this example, the duration of the increased probability lasts about $200 \mathrm{~ms}$ after each spike.

properties of the neurons and its duration varied among units. Flat autocorrelation functions indicated that the probability of spike events was independent of the preceding interval, that is, following the refractory period the occurrence of the next spike was a random process with no tendency to cyclic firing.

Another commonly observed autocorrelation function (67\%, 130/195 striatum; 19\%, 63/331 pallidum) was a single early peak following the initial period of neuronal inactivity (Fig. 8). The early peak signified a period of increased probability for another spike to occur after each spike. For each autocorrelogram, the area of the peak above the distribution mean was measured and computed as a percentage of the area under the mean (Fig. 9). This percentage reflects the increased probability to discharge. In the striatum, burst units had larger initial peaks than non-burst units (Table III, $P<0.001$ ). The relative refractory period measured by the onset time of the initial peak was significantly shorter (Table III, $P<0.001)$ in burst than non-burst units. Thus, burst

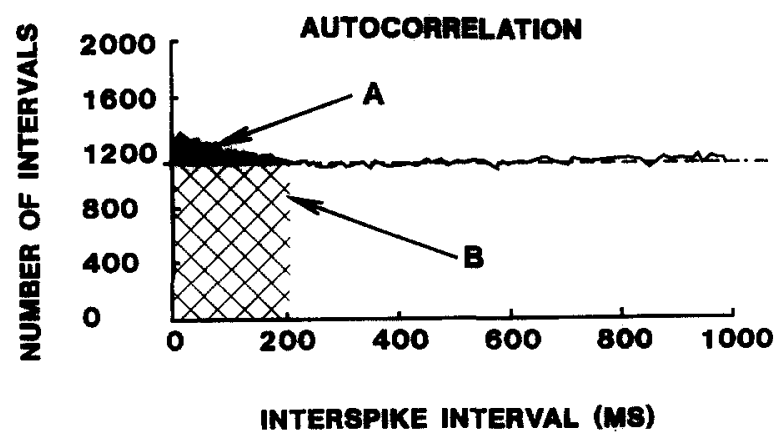

Fig. 9. Early peak autocorrelation of a unit in GPi. The increased probability to discharge was quantified by measuring the area under the curve (shaded area $A$ ) and calculating the percentage of the expected discharge based on the mean discharge rate represented by the area under the mean (cross-hatching $B$ ). The mean of the distribution was calculated by taking the average of the last half of the distribution (dashed line). For clarity, the autocorrelation function is represented by a continuous curve rather than bins. 


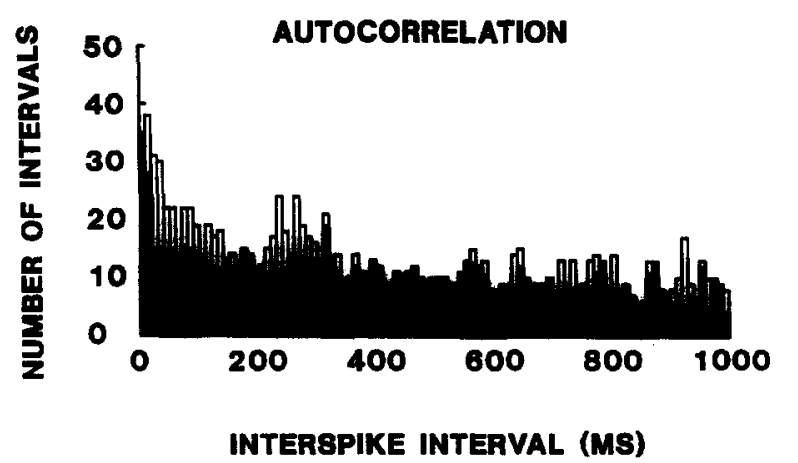

Fig. 10. Autocorrelation of a unit from the putamen. The autocorrelation function of this unit's spike train was computed after the order of the interspike intervals was mixed by random shuffling (filled bins). The autocorrelation function is superimposed on the unshuffled version (unfilled bins). The change due to shuffling indicates that there is a dependence on the serial order of the intervals in this spike train.

units recovered more quickly than non-burst units after each spike. These properties were similar in the putamen and caudate nucleus (Table III).

The types of autocorrelation functions in the globus pallidus were similar to those in the striatum. The major differences were the greater proportion of units with flat autocorrelograms in the pallidum than in the striatum $(81 \%$ vs $33 \%)$. In pallidal units with an early peak in the autocorrelogram, the indices of increased discharge were smaller than in the striatum (Table III). In the pallidum as in the striatum, bursting units made up the greater proportion of units with an early peak in the autocorrelation function. The size of the initial peaks and the length of the refractory period did not differ between the two segments of the globus pallidus.

The autocorrelation analysis was repeated on each spike train after the interspike intervals were shuffled randomly. Since shuffling changes the order but not the composition of intervals in a spike train, a change in the autocorrelation function of the shuffled spike train from

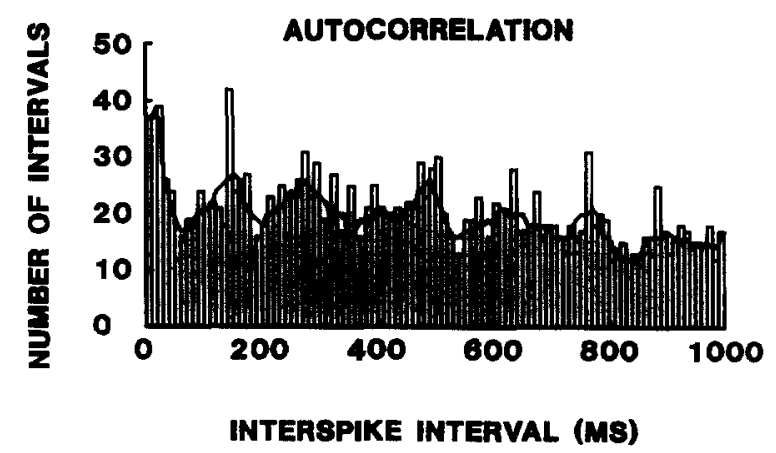

Fig. 11. Autocorrelation function of a putaminal unit. The repetitive rhythmic discharge in the autocorrelation function is highlighted by a superimposed curve. This curve was produced by computing a 5-point running average of the autocorrelation function to smooth the irregularities in bin amplitudes.
BURST UNITS

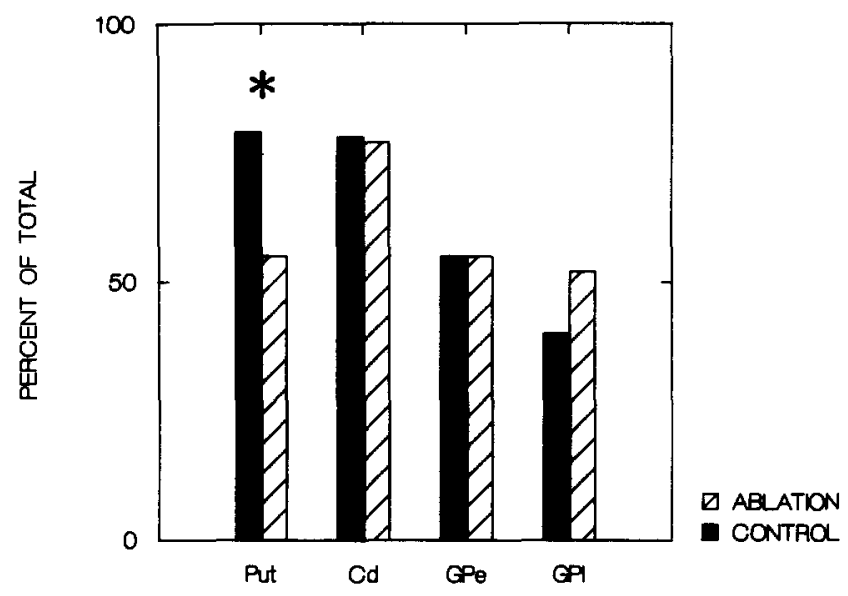

STRUCTURE

Fig. 12. The proportions of units with bursts are represented as a percentage of the total sample. Values obtained after the precentral cortical ablation are indicated by hatched bars. Filled bars are control values. Only the putamen showed a significant change due to the lesion $\left(^{*}\right)$.

its unshuffled counterpart indicates a dependence on the temporal order of interval. The absence of change indicates independence from serial order, that is, the autocorrelation function was determined by composition of interspike intervals alone and not their order. Most units in the striatum and pallidum showed evidence of some dependence on the serial order of their intervals. The autocorrelation functions of shuffled spike trains had similar shapes to those of unshuffled spike trains, but the magnitudes of the initial peaks were usually reduced (Fig. 10). A small proportion of units had autocorrelation functions independent of temporal order.

The autocorrelation function of some units suggested a tendency to oscillation. This was signified by the occurrence of one or more peaks following an initial large peak (Fig. 11). Fourteen percent (28/195) of striatal units, but only $1 \%(4 / 331)$ of pallidal units fell into this category.

\section{Effects of precentral ablation on proportion of bursting units}

Recordings were taken from 520 striatal units and 420 pallidal units in animals with precentral cortical ablation. The proportion of units with bursts in caudate nucleus was unchanged with the ablation (Fig. 12) at $77 \%$ $(54 / 70)$. In contrast, the ablation resulted in a significant decrease $\left(P<0.007, \chi^{2}\right.$ goodness of fit test $)$ in the proportion of putaminal units exhibiting bursts, $55 \%$ (286/520) of the units compared to $79 \%$ in controls (Fig. 
TABLE III

Autocorrelation properties

Increased discharge probability and refractory period. Mean (standard error of mean).

\begin{tabular}{|c|c|c|c|c|c|c|c|c|}
\hline & \multicolumn{4}{|l|}{ Striatum } & \multicolumn{4}{|c|}{ Globus pallidus } \\
\hline & \multicolumn{2}{|c|}{ Caudate nucleus } & \multicolumn{2}{|l|}{ Putamen } & \multicolumn{2}{|c|}{ Internal segment } & \multicolumn{2}{|c|}{ External segment } \\
\hline & Burst & Non-burst & Burst & Non-burst & Burst & Non-burst & Burst & Non-burst \\
\hline Peak size (\% of mean) & $89(13)$ & $38(19)$ & $80(9)$ & $25(5)$ & $48(10)$ & 12 & $46(20)$ & 13 \\
\hline Refractory period (ms) & $1.3(1.0)$ & $10.8(5.3)$ & $1.5(0.5)$ & $9.7(2.7)$ & $0.5(0.2)$ & 1 & $1.3(1.0)$ & 1 \\
\hline$n$ & 24 & 5 & 90 & 11 & 49 & 1 & 12 & 1 \\
\hline
\end{tabular}
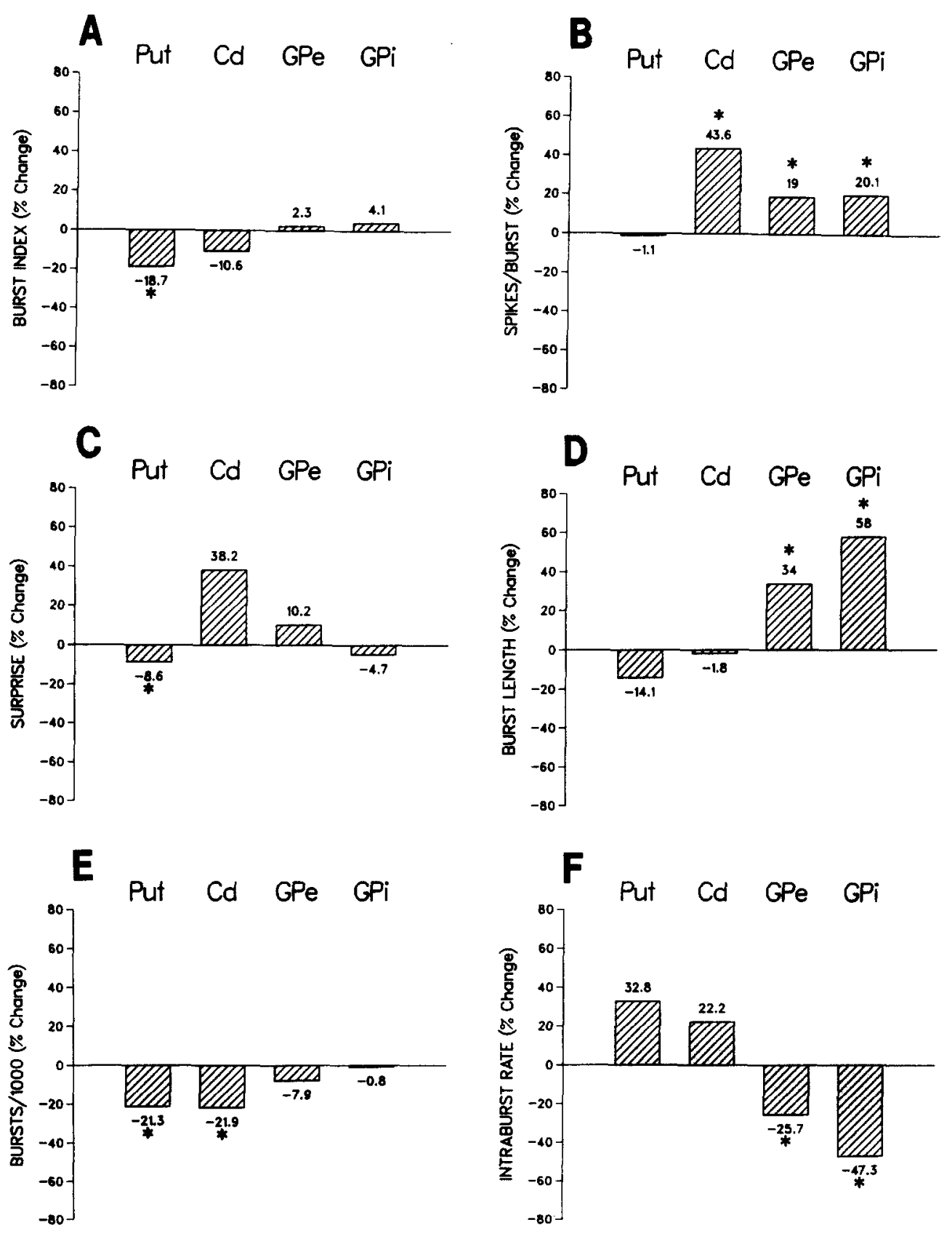

Fig. 13. Post-ablation burst properties. The changes in the quantitative burst properties are illustrated for each structure. The bars represent the percentage increase (positive values) or decrease (negative values) compared to control values. Significant changes are marked with ( ${ }^{*}$ ). The properties illustrated are burst index (A), spikes/burst (B), surprise (C), burst length (D), bursts per 1000 spikes (E), and intraburst rate (F). 
BURST
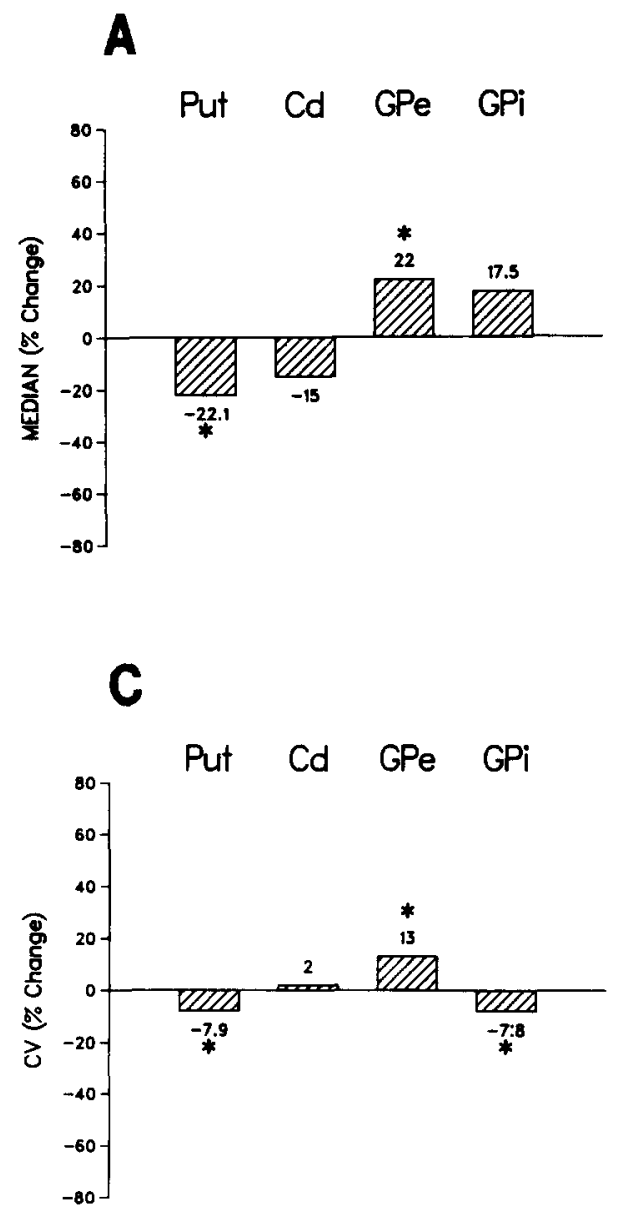

NON-BURST

B

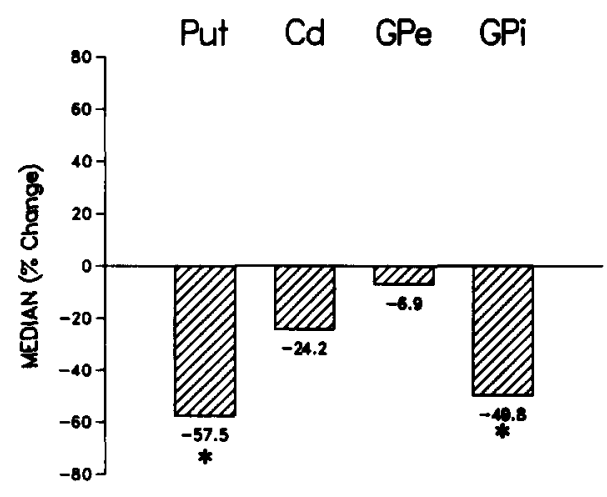

D

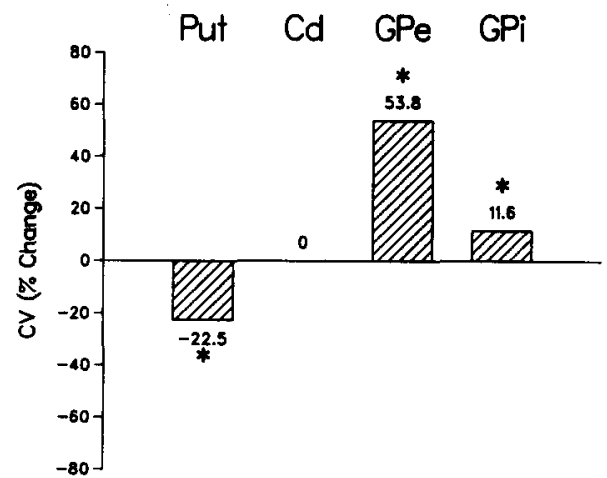

Fig. 14. Changes in burst and non-burst units. The changes in post-ablation median interval sizes in burst units (A) and non-burst units (B) are illustrated. Significant changes are marked with $\left({ }^{*}\right)$. Increases are indicated by positive values and decreases by negative values. The changes in variability as measured by the coefficient of variation are shown for burst units (C) and non-burst units (D). After the ablation, putaminal units have shorter intervals (faster firing) and increased regularity (decreased CV).

12). The proportions of bursting units (Fig. 12) were not affected in either the external segment or internal segment of the globus pallidus.

\section{Post-ablation burst properties}

Bursts in the caudate nucleus differed little from controls. Changes in the burst index (Fig. 13B, $P=$ 0.160 ) and surprise value (Fig. $13 \mathrm{C}, P=0.730$ ) were not significant, but burst rate per 1000 spikes was significantly decreased (Fig. 13E, $P<0.05$ ). Burst length (Fig. 13B, $P=0.436$ ) and intraburst rate (Fig. 13F, $P=0.352$ ) were unchanged. The number of spikes per burst increased (Fig. 13D, $P<0.02$ ). The few putaminal bursts that persisted after the ablation differed from those in controls. Decreased intensity was the most noticeable change (e.g. burst index $=-18.7 \%$, Fig. 13A, $P<$ 0.001 ), and both decreased surprise values (Fig. 13C, $P$ $<0.001$ ) and decreased burst rates per 1000 spikes (Fig.
13E, $P<0.001)$ contributed to this. There was no significant change in burst length (Fig. $13 \mathrm{C}, P=0.124$ ), spikes per burst (Fig. 13D, $P=0.972$ ) or intraburst firing rate (Fig. 13F, $P=0.05$ ).

In the globus pallidus, burst intensity was unchanged after the ablation; the burst index (Fig. 13A; GPe $P=$ 0.331 , GPi $P=0.222$ ), surprise values (Fig. 13C; GPe $P$ $=0.378$, GPi $P=0.817$ ) and bursts per 1000 spikes (Fig. 13E; GPe $P=0.431$, GPi $P=0.141$ ) were not significantly different. There was, however, a significant increase in burst length (Fig. 13B; GPe $P<0.001$, GPi $P<0.001$ ) and spikes per burst (Fig. 13D; GPe $P<0.02$, GPi $P<0.002$ ), and a decrease in intraburst firing rate (Fig. 13F; GPe $P<0.001$, GPi $P<0.001$ ).

After the ablation, a subset of low bursting neurons similar to those in controls was observed. In the caudate nucleus, the size of this subset was unchanged at $15 \%$ of all units. In the putamen, however, the subset was much 
larger after the lesion, increasing to $38 \%$ of all units in the sample.

\section{Effects of precentral ablation on burst units and non-burst units}

In the putamen, both burst and non-burst units showed significant changes in interval sizes and interval variability after the ablation. Median intervals (Fig. 14A, burst $P<0.003$; Fig. 14B, non-burst $P<0.001$ ) and variability decreased (Fig. 14C, burst $P<0.005$; Fig. $14 \mathrm{D}$, non-burst $P<0.001$ ). In the caudate nucleus, the lesion produced no significant change in median interval size (Fig. 14A, burst $P=0.315$; Fig. 14B, non-burst $P=$ 0.065 ) or coefficient of variation (Fig. $14 \mathrm{C}$, burst $P=$ 0.943 ; Fig. $14 \mathrm{D}$, non-burst $P=0.748$ ). The changes in the globus pallidus were complex. After the ablation, in the external segment burst units discharged more slowly (longer intervals) (Fig. 14A, $P<0.03$ ) and had increased variability (Fig. 14C, $P<0.02$ ). Non-burst units exhibited no significant change in rate (Fig. 14B, $P=0.165$ ), but variability increased (Fig. $14 \mathrm{C}, P<0.001$ ). In the internal segment, burst units had no change in rate (Fig. $13 \mathrm{~A}, P=0.371$ ), but variability decreased (Fig. 14C, $P$ $<0.002$ ). Non-burst units had both faster firing rates (Fig. 14B, $P<0.002$ ) and increased variability (Fig. 14D, $P<0.02)$.

\section{Post-ablation autocorrelation properties}

Autocorrelation analysis on spike trains with an adequate number of spikes was possible on $50 \%$ of striatal units (294/590) and $91 \%$ of pallidal units (388/427) after the ablation. The types of autocorrelation functions observed were similar to those in non-ablated animals. In the striatum, the relative proportions of units with a peak in their autocorrelation function did not change. In the putamen, there was a significant decrease in the size of the initial peak in burst units. Non-burst units in the putamen and both burst and non-burst units in the caudate nucleus were not significantly changed by the ablation. There was no effect on the duration of the initial peak in both burst and non-burst units, but the onset time of the peak (or the duration of the relative refractory period) increased in putaminal burst cells after the ablation $(P<0.001)$. In the ablated animals, the proportion of units in the globus pallidus with a flat autocorrelation function dropped significantly $(P<$ 0.001 ) from $81 \%$ to $57 \%$, and conversely, the number of units with an early peak in the autocorrelation function increased. The size of the early peak was unchanged in the globus pallidus in both burst and non-burst units. No other parameter changed after the lesion except for an increased peak duration $(P<0.003)$ and later onset time $(P<0.001)$ in burst units of the external segment.

\section{DISCUSSION}

The principal finding of this study is that bursts of action potentials are responsible for the variability of spontaneous discharge in single units of the basal ganglia. The proportion of bursting units was greatest in the putamen and caudate nucleus followed by the external and then the internal pallidal segments. Because pallidal neurons emit more spikes and bursts per unit of time, they give the impression of intense bursting. In fact, the statistical evaluation demonstrated that bursting contributes more to striatal than to pallidal discharge.

The highest bursting intensities were found in the putamen and caudate nucleus followed by the external and then the internal pallidal segments. The bursting rate per one thousand spikes was the property of bursts that most clearly distinguished these nuclei from each other. For an equal number of spikes, the striatum had higher numbers of bursts than the pallidum. Striatal bursts also had higher surprise values. Bursts in the striatum were generally more conspicuous than bursts in the pallidum with respect to the temporal background of their spike trains. This explains the high degree of variability in striatal discharge.

The bursting properties of single units were not predictable on the basis of average firing rates. In the external pallidal segment, there was no significant difference in median discharge rate between burst and non-burst units. The rate distributions were significantly different in the striatum and internal pallidal segment, although they overlapped. In contrast, discharge variability was an excellent gauge of bursting. Units with high coefficients of variation had more intense bursts than units with low coefficients of variation. The coefficient of variation proved to be a convenient and computationally simple way to identify bursting spike trains in the striatum. The coefficient of variation does not, however, provide information about burst intensity, duration or frequency, which are derived by the surprise method of burst analysis ${ }^{35}$.

The higher overall firing rate in the pallidum than in the striatum was associated with higher intraburst firing rates. All structures studied in the basal ganglia had similar numbers of spikes per burst, but burst durations were different and notably longer in the striatum. Further study of striatal and pallidal bursts in a behavioral context is needed to assess the functional significance of bursts.

In animals with the precentral cortex intact, unit discharge was similar in the caudate nucleus and the putamen in all measures of bursting and spontaneous discharge. Given the similarity of the neuronal architecture in these two nuclei, matching discharge properties are not surprising. The caudate nucleus and putamen 
consist of like populations of neuronal types with analogous configurations of cerebral cortical and subcortical afferents. Due to their prevalence, medium spiny neurons will constitute the majority of the types of neurons sampled. Recording tracks will traverse both striosome and matrix compartments of the striatum; however, these different compartments were not distinguishable in our recordings. This may be due to the fact that the striosome compartment accounts for only $10 \%$ of the striatal volume. Sampling would be biased toward matrix units. A more likely explanation, however, is that a difference in discharge patterns may not be present. Recent studies have demonstrated that many neurophysiological properties of neurons in the two compartments are identical ${ }^{26}$.

In contrast to the striatum, the burst properties of the two pallidal segments were different. The external segment had more intense bursts than the internal segment and the bursts occurred more frequently. These distinctions may reflect differences in neuronal characteristics and/or afferent control mechanisms. Little is known about the membrane properties of pallidal neurons. Morphologically, neurons in the two segments are similar, but afferent pathways do distinguish the two segments. GABAergic striatal afferents to the pallidum are co-localized with substance $P$ in the projection to the internal segment and enkephalin in the projection to the external segment ${ }^{5,23}$. These different co-localized transmitters may lead to different discharge properties in the postsynaptic pallidal neurons. Additionally, the degree of convergence is higher in the internal than the external segment $^{49}$. Nigral projections ${ }^{56}$ to the two segments and dopamine receptors ${ }^{53}$ also distinguish the pallidal segments and may contribute to different discharge patterns.

Our several quantitative assessments indicated that units exhibit a continuum of discharge patterns. Categorizations of units into burst or non-burst types were for convenience and not meant to imply separate morphological types. Because the recording times were relatively short and periods were selected for analysis only during stable quiet behavioral states, spike train properties were stable. A unit might have different properties if it were examined at a different time or in a different behavioral state. Long-term recordings ${ }^{64}$ have demonstrated that individual striatal units can exhibit the full range of variability displayed by the whole population.

A distinct subset of striatal units had low levels of bursting and discharge variability. The units of this subset could not be identified by their discharge rates alone, which overlapped the rest of the sample. Although units in the subset had lower variability than the rest of the sample, they blended in smoothly to the low end of the variability distribution. Whether the units of this subset represent a distinct 'unit type' is unknown. Units with a tonic discharge pattern have been described previously ${ }^{28,}$ ${ }^{29}$, however, these were characterized on the basis of discharge rate and properties associated with the performance of a motor task. In the present study, units in this subset are not likely due to the behavioral state of the animals as a similar proportion of units with regular discharge characteristics was observed in the striatum of animals trained to perform a sensory cued reaching task $^{25}$. The units in this subset could represent the large aspiny cholinergic neurons of the striatum, which comprise about $5 \%$ of striatal neurons ${ }^{27}$. Since cholinergic neurons are larger than medium spiny neurons, the fact that the subset has a larger proportion of units could be explained by larger regions of extracellularly detectable currents ${ }^{36}$ surrounding each cholinergic neuron. Further evidence from intracellular electrophysiological studies ${ }^{63}$ indicates that cholinergic aspiny neurons have spontaneous tonic discharge and membrane properties compatible with the low variability of discharge in units of this category. However, the finding that the putaminal subset enlarged after the cerebral cortical ablation argues against the idea that it represents the discharge of a cholinergic neurons. Rather, the finding suggests that the properties of this subset may be conferred by afferent regulatory mechanisms.

The autocorrelation analysis identified temporal dependencies in intervals of striatal spike trains. In contrast with the striatum, only a small minority of pallidal autocorrelation functions had temporal patterns different than expected from a random spike generating mechanism. The autocorrelation functions revealed that the period of increased probability to discharge following each spike was greater in burst units than non-burst units and the refractory period or recovery time after each spike was shorter in burst units. These findings validate the use of the large early peak as a marker for units with bursts $^{14,64}$. The comparison of shuffled and unshuffled spike trains indicated that the temporal pattern depends not only on the composition of intervals, but also on the serial order of intervals. It is possible that repeating patterns of spikes ${ }^{1,8,9}$ may contribute to the dependence on serial order. In other systems, serial dependencies vary with the driven state of the neurons $\mathrm{s}^{51,61}$ and have larger numbers of units with periodicities. Relatively few basal ganglia units with a periodic discharge were observed.

Ablation of the precentral area of cerebral cortex markedly reduced bursting in the putamen. Units that continued to burst had lower surprise values and fewer bursts for the same number of spikes. In contrast, bursting was only minimally changed in the caudate nucleus. These findings explain the changes in discharge variability after the lesion ${ }^{3}$.

Increased putaminal firing rates were observed in both 
burst and non-burst units, which was surprising given the fact that the cortical input is thought to be excitatory. This finding suggests that mechanisms controlling discharge rate and the bursting pattern are to some extent independent. Afferent control mechanisms are more important to the regulation of temporal discharge pattern than discharge rate. Moreover, the properties of the cortico-striatal network may determine the characteristic discharge patterns and the post-ablation discharge patterns may be due to changes in network configuration and interactions. Multiple levels of convergence and divergence throughout the network suggest that the regulation of discharge is not a simple combination of the excitatory or inhibitory transmitters in the pathway. Evidence from other studies supports this contention. Lesioning striatal efferents to the pallidum affects the pattern of discharge more than the rate ${ }^{54}$. Disturbances in other circuits of the basal ganglia also have a significant impact on discharge pattern ${ }^{14.45}$. Together, these studies suggest that discharge rate in a neuron may to some extent be self-regulating and that information transfer in these networks may depend more on pattern than rate.

The ablation undoubtedly triggers compensatory mechanisms. Regulation of receptors and morphological plasticity are potential mechanisms ${ }^{15.24,42,43,59,61}$. At the membrane level, changes in membrane potentials, ion channel properties and conductance properties ${ }^{7.17}$ may alter the characteristics of neuronal responses to intact afferents. Since the ablation-induced changes were apparent in the first recording sessions after afferent lesions and showed no recovery over the course of the study ${ }^{3}$, longer recovery times may be necessary to observe the results of the compensatory mechanisms that operate at the membrane level. At the network level, changes could appear immediately. Simulations of afferent control systems indicate that reducing the number of inputs increases variability ${ }^{55}$. This suggests that the impact of a restricted cerebral cortex lesion on the number of inputs a striatal neuron receives may be small, perhaps due to the enormous convergence on individual neurons ${ }^{49}$ and the fact that cortical terminals may be electrically isolated on spines ${ }^{16}$. Simulations also indicate that the coefficient of variation properties depend on the coefficient of variation properties of input neurons ${ }^{55}$. Variability di-

\section{REFERENCES}

1 Abeles, M. and Gerstein, G.L., Detecting spatiotemporal firing patterns among simultaneously recorded single neurons, $J$. Neurophysiol., 60 (1988) 909-924.

2 Albin, R.L., Young, A.B. and Penney, J.B., The functional anatomy of basal ganglia disorders, Trends Neurosci., 12 (1989) 366-375.

3 Aldridge, J.W., Gilman, S. and Dauth, G.W., Spontaneous neuronal unit activity in the primate basal ganglia and the effects minishes as the variability of inputs decreases ${ }^{55}$. Thus, intact afferents may have lower variability levels after the lesion than before.

Information processing mechanisms that utilize the temporal pattern of spike discharge have advantages. At the network level, the computational properties of neuronal networks may be enhanced ${ }^{20}$. The output of a network can be varied by the ability of some of its constituents to produce bursts ${ }^{38}$, suggesting that burst modulation could lead to multiple network states. At the synaptic level, temporal discharge pattern is known to modulate neuronal output. Synaptic release from a regular discharge pattern is less compared to a bursting sequence with the same number of spikes ${ }^{13}$. The differential release of co-localized transmitters may depend on the discharge pattern ${ }^{62}$. Long-term network connectivity may also depend on discharge pattern since phasic activity influences the functional architecture of synaptic connections ${ }^{42}$ by favoring active pathways over inactive ones.

The results of the present study suggest that the striatum uses an irregular pattern of bursting as a mechanism for information transfer. Compared to ratecoding, a burst-dependent mechanism would be faster and more flexible in units with normally slow discharge rates. In contrast with the striatum, the pallidum has a regular discharge pattern although a significant proportion of burst units was detected in both segments. Because of the high discharge rates in many pallidal units, modulating discharge rates up or down would provide an efficient and fairly rapid mechanism to encode and transfer information. The rate of pallidal discharge during learned movement in trained animals is highly correlated to the amplitude and/or velocity of movements ${ }^{19}$ or the phase of a movement ${ }^{6}$, suggesting that discharge rate may encode the parameters of movement. The use of one mechanism does not preclude the use of other mechanisms, even ones operating simultaneously.

Acknowledgements. The authors acknowledge the technical assistance of: M. Gallagher, D. Jaeger, M. Lesperance, S. Ruger, R. Sachdev, M. Samson, and J. Walden and helpful comments from Drs. G.W. Dauth and R. Albin. This work was supported in part by NIH Grants NS19613 and NS07222 and by a grant from the United Cerebral Palsy Research and Education Foundation, Inc.

of precentral cerebral cortical ablations, Brain Research, 516 (1990) 46-56.

4 Aldridge, J.W., Walden, J.L. and Gilman, S., Enhancing high speed digitization of single unit neuronal activity on a microcomputer using a hybrid software-hardware technique, J. Neurosci. Methods, 28 (1989) 205-208.

5 Anderson, K.D. and Reiner, A., Extensive co-occurrence of substance-P and dynorphin in striatal projection neurons - an evolutionarily conserved feature of basal ganglia organization, $J$. Comp. Neurol., 295 (1990) 339-369. 
6 Anderson, M.E., Discharge patterns of basal ganglia neurons during active maintenance of postural stability and adjustment to chair tilt, Brain Research, 143 (1977) 325-338.

7 Bargas, J., Galarraga, E. and Aceves, J., An early outward conductance modulates the firing latency and frequency of neostriatal neurons of the rat brain, Exp. Brain Res., 75 (1989) 146-156.

8 Dayhoff, J.E. and Gerstein, G.L., Favored patterns in spike trains. I. Detection, J. Neurophysiol., 49 (1983) 1334-1348.

9 Dayhoff, J.E. and Gerstein, G.L., Favored patterns in spike trains. II. Application, J. Neurophysiol., 49 (1983) 1349-1363.

10 DeLong, M.R., Activity of pallidal neurons during movement, J. Neurophysiol., 34 (1971) 414-427.

11 Denny-Brown, D., The Cerebral Control of Movement, Liverpool University Press, Liverpool, 1966.

12 Divac, I., Fonnum, F. and Storm-Mathisen, J., High affinity uptake of glutamate in terminals of corticostriatal axons, Nature, 266 (1977) 377-378.

13 Dutton, A. and Dyball, R.E., Phasic firing enhances vasopressin release from the rat neurohypophysis, J. Physiol., 290 (1979) 433-440.

14 Filion, M., Effects of interruption of the nigrostriatal pathway and of dopaminergic agents on the spontaneous activity of globus pallidus neurons in the awake monkey, Brain Research, 178 (1979) 425-441.

15 Finger, S. and Almli, C.R., Brain damage and neuroplasticity: mechanism of recovery or development?, Brain Res. Rev., 10 (1985) 177-186.

16 Freund, T.F., Powell, J.F. and Smith, A.D., Tyrosine hydroxylase-immunoreactive boutons in synaptic contact with identified striatonigral neurons, with particular reference to dendritic spines, Neuroscience, 13 (1985) 1189-1216.

17 Galarraga, E., Bargas, J., Sierra, A. and Aceves, J., The role of calcium in the repetitive firing of neostriatal neurons, Exp. Brain Res., 75 (1989) 157-168.

18 Garcia-Rill, E., Hull, C.D., Levine, M.S. and Buchwald, N.A., The spontaneous firing patterns of forebrain neurons. IV. Effects of bilateral and unilateral frontal cortical ablations on firing of caudate, globus pallidus and thalamic neurons, Brain Research, 165 (1979) 23-36.

19 Georgopoulos, A.P., DeLong, M.R. and Crutcher, M.D., Relations between parameters of step-tracking movements and single cell discharge in the globus pallidus and subthalamic nucleus of the behaving monkey, J. Neurosci., 3 (1983) 15861598.

20 Getting, P.A., Emerging principles governing the operation of neural networks, Ann. Rev. Neurosci., 12 (1989) 185-204.

21 Gilman, S., Lieberman, J.S. and Marco, L.A., Spinal mechanisms underlying the effects of unilateral ablation of areas 4 and 6 in monkeys, Brain, 97 (1974) 49-64.

22 Graybiel, A.M., Neurochemically specified subsystems in the basal ganglia. In Functions of the Basal Ganglia, Ciba Foundation Symposium 107, Pitman, London, 1984, pp. 114-149.

23 Graybiel, A.M., Neuropeptides in the basal ganglia. In J.B. Martin and J.D. Barchas (Eds.), Neuropeptides in Neurologic and Psychiatric Disease, Raven Press, New York, 1986, pp. 135-161.

24 Ingham, C.A., Hood, S.H. and Arbuthnott, G.W., Spine density on neostriatal neurones changes with 6-hydroxydopamine lesions and with age, Brain Research, 503 (1989) 334-338.

25 Jaeger, D., Aldridge, J.W. and Gilman, S., Basal ganglia unit activity in relation to movement preparation, unpublished results in preparation.

26 Kawaguchi, Y., Wilson, C.J. and Emson, P.C., Intracellular recording of identified neostriatal patch and matrix spiny cells in a slice preparation preserving cortical inputs, J. Neurophysiol., 62 (1989) 1052-1068.

27 Kemp, J.M. and Powell, T.P., The structure of the caudate nucleus of the cat: light and electron microscopy, Philos. Trans. R. Soc. Lond. Biol. Sci., 262 (1971) 383-401.
28 Kimura, M., The role of primate putamen neurons in the association of sensory stimuli with movement, Neurosci. Res., 3 (1986) 436-443.

29 Kimura, M., Rajkowski, J.R. and Evarts, E., Tonically discharging putamen neurons exhibit set-dependent responses, Proc. Natl. Acad. Sci. U.S.A., 81 (1984) 4998-5001.

$30 \mathrm{Kita}$ H., Kita, T. and Kitai, S.T., Active membrane properties of rat neostriatal neurons in an in vitro slice preparation, Exp. Brain Res., 60 (1985) 54-62.

31 Kita, H., Kita, T. and Kitai, S.T., Regenerative potentials in rat neostriatal neurons in an in vitro slice preparation, Exp. Brain Res., 60 (1985) 63-70.

$32 \mathrm{Kita}, \mathrm{H}$. and Kitai, S.T., Glutamate decarboxylase immunoreactive neurons in rat neostriatum: their morphological type and populations, Brain Research, 447 (1988) 346-359.

33 Kolb, B., Day, J., Gibb, R. and Whishaw, I.Q., Recovery from early cortical lesions in rats. 6. Cortical noradrenaline, cortical thickness, and development of spatial learning after frontal lesions or hemidecortications, Psychobiology, 17 (1989) 370376.

34 Kolb, B., Zaborowski, J. and Whishaw, I.Q., Recovery from early cortical damage in rats. 5. Unilateral lesions have different behavioral and anatomical effects than bilateral lesions, Psychobiology, 17 (1989) 363-369.

35 Legendy, C.R. and Salcman, M., Bursts and recurrences of bursts in the spike trains of spontaneously active striate cortex neurons, J. Neurophysiol., 53 (1985) 926-939.

36 Lemon, R., Methods for Neuronal Recording in Conscious Animals, IBRO Handbook Series: Methods in Neuroscience, Wiley, Chichester, 1984.

37 Lemon, R.N. and Mantel, G.W.H., The influence of changes in discharge frequency of corticospinal neurones on hand muscles in the monkey, J. Physiol., 413 (1989) 351-378.

38 Marder, E. and Nusbaum, M.P., Peptidergic modulation of the motor pattern generators in the stomatogastric ganglion. In Perspectives in Neural Systems and Behavior, 1989, pp. 73-91.

39 McGeer, E.G., Staines, W.A. and McGeer, P.L., Neurotransmitters in the basal ganglia, Can. J. Neurol. Sci., 11 (1984) 89-99.

40 McGeer, P.L., McGeer, E.G., Scherer, U. and Singh, K., A glutamatergic cortico-striatal path?, Brain Research, 128 (1977) 369-373.

41 Moore, G.P., Perkel, D.H. and Segundo, J.P., Statistical analysis and functional interpretation of neuronal spike data, Ann. Rev. Physiol., 15 (1966) 493-522.

42 Nelson, P.G., Yu, C., Fields, R.D. and Neale, E.A., Synaptic connections in vitro - modulation of number and efficacy by electrical activity, Science, 244 (1989) 585-587.

43 Nothias, F., Wictorin, K., Isacson, O., Björklund, A. and Peschanski, M., Morphological alteration of thalamic afferents in the excitotoxically lesioned striatum, Brain Research, 461 (1988) 349-354.

44 Pan, H.S., Frey, K.A., Young, A.B. and Penney Jr., J.B., Changes in $\left[{ }^{3} \mathrm{H}\right]$ muscimol binding in substantia nigra, entopeduncular nucleus, globus pallidus, and thalamus after striatal lesions as demonstrated by quantitative receptor autoradiography, J. Neurosci., 3 (1983) 1189-1198.

45 Pan, H.S. and Walters, J.R., Unilateral lesion of the nigrostriatal pathway decreases the firing rate and alters the firing pattern of globus pallidus neurons in the rat, Synapse, 2 (1988) 650-656.

46 Parmiggiani, F. and Stein, R.B., Nonlinear summation of contractions in cat muscles. II. Later facilitation and stiffness changes, J. Gen. Physiol., 78 (1981) 295-311.

47 Penney Jr., J.B. and Young, A.B., Speculations on the functional anatomy of basal ganglia disorders, Ann. Rev. Neurosci., 6 (1983) 73-94.

48 Penny, G.R., Afsharpour, S. and Kitai, S.T., The glutamate decarboxylase-, leucine enkephalin-, methionine enkephalin-, and substance P-immunoreactive neurons in the neostriatum of the rat and cat: evidence for partial population overlap, 
Neuroscience, 17 (1986) 1011-1045.

49 Percheron, G., Yelnik, J. and Francois, C., A Golgi analysis of the primate globus pallidus. III. Spatial organization of the striato-pallidal complex, J. Comp. Neurol., 227 (1984) 214-227.

50 Perkel, D.H., Gerstein, G.L. and Moore, G.P., Neuronal spike trains and stochastic point processes. I. The single spike train, Biophys. J., 7 (1967) 391-418.

51 Poggio, G.F. and Viernstein, L.J., Time series analysis of impulse sequences of thalamic somatic sensory neurons, $J$. Neurophysiol., 27 (1964) 517-545.

52 Pubols, B.H., Haring, J.H. and Rowinski, M.J., Patterns of resting discharge in neurons of the raccoon main cuneate nucleus, J. Neurophysiol., 61 (1989) 1131-1141.

53 Richfield, E.K. Young, A.B. and Penney, J.B., Comparative distributions of dopamine D-1 and D-2 receptors in the cerebral cortex of rats, cats, and monkeys, J. Comp. Neurol., 286 (1989) 409-426.

54 Sachdev, R.N.S., Gilman, S. and Aldridge, J.W., Effects of excitotoxic striatal lesions on single unit activity in globus pallidus and entopeduncular nucleus of the cat, Brain Research, 501 (1989) 295-306.

55 Segundo, J.P., Perkel, D.H., Wyman, H., Hegstad, H. and Moore, G.P., Input-output relations in computer-simulated nerve cells, Kybernetik, 4 (1968) 157-171.

56 Smith, Y., Lavoie, B., Dumas, J. and Parent, A., Evidence for a distinct nigropallidal dopaminergic projection in the squirrel monkey, Brain Research, 482 (1989) 381-386.

57 Tremblay, L., Filion, M. and Bedard, P.J., Responses of pallidal neurons to striatal stimulation in monkeys with MPTP-induced parkinsonism,, Brain Research, 498 (1989) 17-33.

58 Vibert, J.F. and Costa, J., Spike separation in multiunit records: a multivariate analysis of spike descriptive parameters, EEG Clin. Neurophysiol., 47 (1979) 172-182.

59 Waddington, J.L. and Cross, A.J., Denervation supersensitivity in the striatonigral GABA pathway, Nature, 276 (1978) 618-620.

60 Waddington, J.L. and Cross, A.J., Characterization of denervation supersensitivity in the striatonigral GABA pathway of the kainic acid-lesioned rat and in Huntington's disease, Brain Res. Bull., 5 (1980) 825-828.

61 Werner, G. and Mountcastle, V.B., The variability of central neural activity in a sensory system, and its implications for the central reflection of sensory events, J. Neurophysiol., 26 (1963) 958-977.

62 Whim, M.D. and Lloyd, P.E., Frequency-dependent release of peptide cotransmitters from identified cholinergic motor neurons in aplysia, Proc. Natl. Acad. Sci. U.S.A., 86 (1989) 9034-9038.

63 Wilson, C.J., Chang, H.T. and Kitai, S.T., Firing patterns and synaptic potentials of identified giant aspiny interneurons in the rat neostriatum, J. Neurosci., 10 (1990) 508-519.

64 Wilson, C.J. and Groves, P.M., Spontaneous firing patterns of identified spiny neurons in the rat neostriatum, Brain Research, 220 (1981) 67-80.

65 Young, A.B., Bromberg, M.B. and Penney, Jr., J.B., Decreased glutamate uptake in subcortical areas deafferented by sensorimotor cortical ablation in the cat, $J$. Neurosci., 1 (1981) 241-249. 